\title{
Flatness control of a crane
}

\author{
H. Souilem ${ }^{1}$, H. Mekki ${ }^{2}$ and N. Derbel ${ }^{1}$ \\ Control, Energy and Management Lab (CEM Lab) \\ ${ }^{1}$ National School of Engineers of Sfax BP.W, 3038, Sfax-Tunisia \\ ${ }^{2}$ National School of Engineers of Sousse
}

Email address:

haifa.souilem@gmail.com (H. Souilem); hassen.mekki@eniso.rnu.tn (H. Mekki); nabil.derbel@ieee.org (N. Derbel)

To cite this article:

H. Souilem ,H. Mekki and N. Derbel. Flatness Control of a Crane. Automation, Control and Intelligent systems. Vol. 1, No. 1, 2013, pp. 1-7. doi: $10.11648 /$ j.acis.20130101.11

\begin{abstract}
The aim of this work is to propose a flatness control of a crane detailing adopted mechanisms and approaches in order to be able to control this system and to solve problems encountered during its functioning. The control objective is the sway-free transportation of the crane's load taking the commands of the crane operator into account. Based on the mathematical model linearizing and stabilizing control laws for the slewing and luffing motion are derived using the input/output linearization approach. The method allows for transportation of the payload to a selected point and ensures minimisation of its swings when the motion is finished. To achieve this goal a mathematical model of the control system of the displacement of the payload has been constructed. A theory of control which ensures swing-free stop of the payload is presented. Selected results of numerical simulations are shown. At the end of this work, a comparative study between the real moving and the desired one has been presented.
\end{abstract}

Keywords: Crane, Flatness Control, Path Planning, Path Tracking

\section{Introduction}

Flatness-based control techniques have been developed and applied in many industrial processing with a great success in solving planning and tracking problems of reference trajectories such as thermal process control [15], motors control [1], chemical reactor control [16], crane control [10] etc... This theory was introduced in 1992 by M. Fliess and al. [6].

The existence of a variable called a flat output permits to define all other system variables. The dynamics of such process can be then deduced without solving differential equations. Therefore, it is possible to express the state, as well as the input and the output system, as differential functions of the flat output [6] [11].

Conventionally, it is difficult to resolve the path planning problem due to the necessity to solve the differential system equations from the initial conditions to obtain the solution at the final time. In the case of flat systems, this problem can be solved easily without approximation and without solving differential systems equations. Indeed, flatness property ensures the existence of a flat output which allows the parameterization of all system variables as a function of finite number of its derivatives.

The goal of this work is to solve problems encountered during the motion of the load using the technique known by control by fatness whose main objective is to attenuate the undesirable swings of the load [7], [9], [13]. In fact, the differential flatness has been introduced by Fliess and al. [5] in 1995. The states and the in puts of the flat system can be expressed in function of the particular out puts and their successive derivatives. We can find a lot of the literature uses a linear approaches [3], [8], [19] or an approaches of optimal control [12], [20]. Also, several methods are proposed in [9] and [14] in order to decrease the oscillations created by the outsides disruptions. Authors of [2] and [4] use energizing techniques by exploiting the fact that a crane can be identified to a pendulum if we fixes the length of the vertical cable bound to the load. Other techniques can be useful: technique of in put / out put linearization, technique of in put / state linearization, but these techniques present several problems which are so difficult to solve it. We here interested to exploit the concept of the flatness in order to control the system crane: in section 2, we present the dynamic model of the crane. In section 3 , the crane is modeled by a flat system. Section 4 deals with flatness and linearization. Section 5 deals with flatness and trajectories generation. Finally, in section 6, we present the flatness and the tracking of trajectory. 


\section{Formulation of the Problem}

The most of the weight handling equipments use ropes and winches in order to displace the load. This system of raising went up on a mechanical structure with one or two articulations.

Among these weight handling equipments, we consider the crane system which its characteristics are the following:

- A load of mass $M$ which its coordinates are: $(\xi, \zeta)$

A weight handling equipment compound a rope, pulleys and winches. The motors manipulating the winches are supposed to be controlled by two couples: $T_{1}$ and $T_{2}$.

- A mechanical structure entirely articulate on which is fixed the winches of coil of the ropes.

- A rail breaks the movement of the pulley.

The tabular diagram of the crane is given by the following figure: [17]

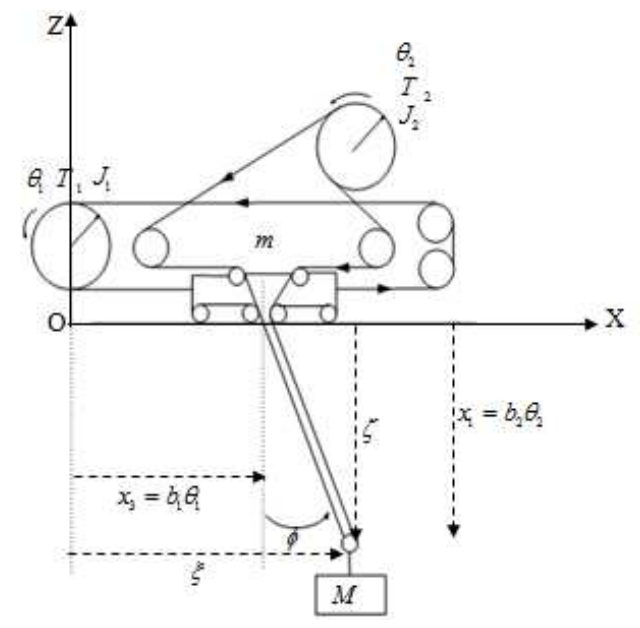

Figure 1. Crane in dimension 2.

\subsection{Model of the crane}

The dynamic model of the crane can be given by: [17]

$$
\begin{aligned}
& T_{1}=\left[J_{1}+(M+m) b_{1}^{2}\right] \ddot{\theta}_{1}+M b_{1} b_{2} \ddot{\theta}_{2} \sin \phi \\
& +M b_{1} b_{2}\left[2 \dot{\theta}_{2} \dot{\phi} \cos \phi-\theta_{2}(\dot{\phi})^{2} \sin \phi\right] \\
& +M b_{1} b_{2} \ddot{\phi} \theta_{2} \cos \phi \\
& T_{2}=\left(J_{2}+M b_{2}^{2}\right) \ddot{\theta}_{2}-M b_{2}^{2} \theta_{2}(\dot{\phi})^{2} \\
& +M b_{1} b_{2} \ddot{\theta}_{1} \sin \phi-M g b_{2} \cos \phi \\
& b_{1} \ddot{\theta}_{1} \cos \phi+b_{2} \theta_{2} \ddot{\phi}+2 b_{2} \dot{\theta}_{2} \dot{\phi}+g \sin \phi=0
\end{aligned}
$$

The geometric constraints between the coordinates of the wagon and the load:

$$
\xi=x_{3}+x_{1} \sin \phi
$$

$$
\zeta=x_{1} \cos \phi
$$

with,

$$
x_{1}=b_{2} \theta_{2}^{\prime} x_{3}=b_{1} \theta_{1}
$$

thus, we gets:

$$
\begin{aligned}
& \left(\begin{array}{lll}
\frac{J_{1}}{b_{1}}+(M+m) b_{1} & M \cdot b_{1} \sin \phi & M \cdot b_{1} x_{1} \cos \phi \\
M \cdot b_{2} \sin \phi & \frac{J_{2}}{b_{2}}+M \cdot b_{2} & 0 \\
\cos \phi & 0 & x_{1}
\end{array}\right)\left(\begin{array}{l}
\ddot{x_{3}} \\
\ddot{x_{1}} \\
\ddot{\phi}
\end{array}\right)= \\
& \left(\begin{array}{l}
T_{1}-M b_{1}\left(2 \dot{x_{1}} \dot{\phi} \cos \phi-x_{1} \dot{\phi}^{2} \sin \phi\right) \\
T_{2}+M g b_{2} \cos \phi+M \cdot b_{2} x_{1} \dot{\phi}^{2} \\
-g \sin \phi-2 \dot{x_{1} \dot{\phi}}
\end{array}\right)
\end{aligned}
$$

So an explicit representation of the system can be obtained $\left(x_{3}, \dot{x}_{3}, x_{1}, \dot{x}_{1}, \emptyset, \dot{\emptyset}\right)$.

\section{Flat Systems}

Recent research in trajectory tracking control has focused on systems with a property known as differential flatness. A nonlinear system $\dot{x}=f(x, u)$ is differentially flat if an output $y$ can be found such that the states $x$ and inputs $u$ can be expressed in terms of $y$ and a finite number of its derivatives [4]. A benefit of flat systems is that flat outputs can follow arbitrary trajectories $y_{d}(t)$ provided that the trajectory is sufficiently smooth. For this system we can choose the position of the load denoting by $(\xi, \zeta)$, and we verify if it presents a flat output, that is to say, verify that all variables and all controls of the system can be expressed in function of this chosen output. According to the model (1), we can see that the third equation can be got by using the derivative in order two of $\xi$ and $\zeta$, more precisely, by using the following expression:

$$
\ddot{\xi} \cos \phi-\ddot{\zeta} \sin \phi=-g \sin \phi
$$

We obtain:[18]

$$
\begin{gathered}
x_{3}=\xi-\frac{\ddot{\xi} \zeta}{\ddot{\zeta}-g}, x_{1}^{2}=\zeta^{2}+\left(\frac{\ddot{\xi} \zeta}{\ddot{\xi}-g}\right)^{2}, \\
\phi=\arctan \left(\frac{\ddot{\xi}}{\ddot{\xi}-g}\right)
\end{gathered}
$$




$$
\begin{aligned}
& T_{1}=M b_{1}\left(2 \dot{x_{1}} \dot{\phi} \cos \phi-x_{1} \dot{\phi}^{2} \sin \phi\right)+\left(\frac{J_{1}}{b_{1}}+\right. \\
& \left.(M+m) b_{1}\right) \ddot{x_{3}}+M b_{1} \sin \phi \ddot{x_{1}}+M b_{1} x_{1} \cos \phi \ddot{\phi}
\end{aligned}
$$

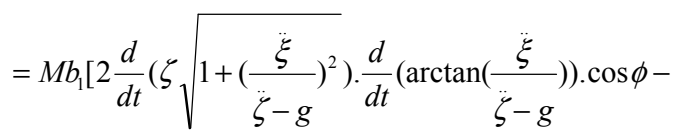

$$
\begin{aligned}
& \left.x_{1}\left(\frac{d}{d t}\left(\arctan \left(\frac{\ddot{\xi}}{\check{\zeta}-g}\right)\right)\right)^{2} \sin \phi\right]+\left(\frac{J_{1}}{b_{1}}+(M+m) b_{1}\right) .
\end{aligned}
$$

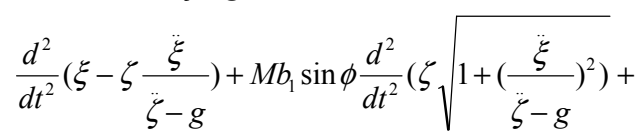

$$
\begin{aligned}
& M b_{1} x_{1} \cos \phi \frac{d^{2}}{d t^{2}}\left(\arctan \left(\frac{\ddot{\xi}}{\ddot{\zeta}-g}\right)\right) . \\
& T_{2}=-M g b_{2} \cos \phi-M b_{2} x_{1} \dot{\phi}^{2}+M b_{2} \sin \phi \ddot{x}_{3}+ \\
& \left(\frac{J_{2}}{b_{2}}+M b_{2}\right) \ddot{x_{1}} \text {. } \\
& =-M g b_{2} \cos \phi-M b_{2} x_{1}\left(\frac{d}{d t}\left(\arctan \left(\frac{\ddot{\xi}}{\ddot{\zeta}-g}\right)\right)\right)^{2}+ \\
& M b_{2} \sin \phi \frac{d^{2}}{d t^{2}}\left(\xi-\zeta \frac{\ddot{\xi}}{\ddot{\zeta}-g}\right)+\left(\frac{J_{2}}{b_{2}}+M b_{2}\right) \\
& \frac{d^{2}}{d t^{2}}\left(\zeta \sqrt{\left.1+\left(\frac{\ddot{\xi}}{\ddot{\zeta}-g}\right)^{2}\right) .}\right.
\end{aligned}
$$

It is easy to see that all variables of the system denoting by $\left(x_{3}, x_{1}, \xi, \zeta, T_{1}, T_{2}\right)$ can be expressed in function of $\xi$ and $\zeta$ (the coordinates of the load) and of their derivatives until the order 4 , this result is compatible with the principle of flatness.

\section{Flatness and linearization}

We are interested in this paragraph to appear a dynamic endogenous feedback.

In fact, by using expressions in subsection 3 , we can put

$$
\begin{gathered}
\xi^{(4)}=v_{1} \\
\zeta^{(4)}=v_{2}
\end{gathered}
$$

Then, the dynamic endogenous feedback can be calculated by identifying the derivatives of $\xi$ and $\zeta$ until order 4 with their expressions in terms of inputs $T_{1}$ and $T_{2}$.

According to the equations in subsection 3 and by making the change of the control, we obtain:

$$
\begin{aligned}
& w_{1}=\frac{1}{H}\left(b_{2} J_{1} T_{2}+\sin \varphi b_{1} J_{2} T_{1}-\right. \\
& \cos \varphi g J_{1} J_{2}+b_{1}^{2} b_{2} m T_{2}-\cos \varphi g m J_{2} b_{1}^{2}- \\
& -x_{1} \dot{\varphi} J_{1} J_{2}-x_{1} \dot{\varphi}^{2} b_{1}^{2} m J_{2}
\end{aligned}
$$

with,

$$
\begin{aligned}
& H=J_{1} J_{2}+J_{1} M b_{2}^{2}+b_{1}^{2} M J_{2}+b_{1}^{2} m J_{2}+ \\
& b_{1}^{2} m M b_{2}^{2}-\cos \phi^{2} M b_{1}^{2} J_{2}
\end{aligned}
$$

Then, we will have:

$$
\begin{aligned}
& \ddot{\xi}=w_{1} \sin \phi \\
& \ddot{\zeta}=w_{1} \cos \phi+g
\end{aligned}
$$

By deriving again $\xi$ and $\zeta$ two times and using expressions in subsection (4),

we have:

$$
\begin{aligned}
& \xi^{(4)}=\ddot{w}_{1} \sin \varphi+2 \dot{w}_{1} \dot{\varphi} \cos \varphi-w_{1} \dot{\varphi}^{2} \sin \varphi+ \\
& w_{1} w_{2} \cos \varphi=v_{1} \\
& \zeta^{(4)}=\ddot{w_{1}} \cos \varphi-2 \dot{w}_{1} \dot{\varphi} \sin \varphi-w_{1} \dot{\varphi}^{2} \cos \varphi- \\
& w_{1} w_{2} \sin \varphi=v_{2}
\end{aligned}
$$

By reversing this linear system in relation to $w_{1}$ and $w_{2}$, we will have:

$$
\begin{aligned}
& \ddot{w}_{1}=\sin \theta \cdot v_{1}+\cos \theta \cdot v_{2}+w_{1} \dot{\theta}^{2} \\
& w_{2}=\frac{1}{w_{1}}\left(\cos \theta \cdot v_{1}-\sin \theta \cdot v_{2}-2 \dot{w_{1}} \dot{\theta}\right)
\end{aligned}
$$

By this way, we construct a dynamic endogenous feedback by introducing a compensator whose state is given by $\left(w_{1}, \dot{w}_{1}, w_{2}\right)$

Then the problem that remains to solve consists to the generation of trajectory leaving from an initial position arriving to a final position.

\section{Flatness and Generation of Trajectories}

We suppose that we want to bring the load from the position of departure $\left(\xi_{i}, \zeta_{i}\right)$ at the instant $t_{i}$, without moving, to the final position $\left(\xi_{f}, \zeta_{f}\right)$ at the instant $t_{f}$, also without moving, and to make it pass by the point denoting by: $\left(\frac{\xi_{f}-\xi_{i}}{2}, 2 \zeta_{f}-\zeta_{i}\right)$ which must be the maximum of this curve between $\xi_{i}$ and $\xi_{f}$. 
This trajectory $\zeta(\xi)$ must verify the four conditions denoting by:

$$
\begin{aligned}
& \zeta\left(\xi_{i}\right)=\zeta_{i}, \zeta\left(\xi_{f}\right)=\zeta_{f}, \zeta\left(\frac{\xi_{f}-\xi_{i}}{2}\right)=2 \zeta_{f}-\zeta_{i} \\
& \frac{d \zeta}{d \xi}\left(\frac{\xi_{f}+\xi_{i}}{2}\right)=0
\end{aligned}
$$

With the constraint $\frac{d^{2} \zeta}{d^{2} \xi}\left(\frac{\xi_{f}+\xi_{i}}{2}\right)<0$ in order to have a local maximum in this point.

We can choose the polynomial of third degree in $\xi$ denoting by:

$$
\begin{aligned}
& \zeta(\xi)=\zeta_{i}+\left(\zeta_{f}-\zeta_{i}\right)\left(\frac{\xi-\xi_{i}}{\xi_{f}-\xi_{i}}\right)\left(9-12\left(\frac{\xi-\xi_{i}}{\xi_{f}-\xi_{i}}\right)+\right. \\
& \left.4\left(\frac{\xi-\xi_{i}}{\xi_{f}-\xi_{i}}\right)^{2}\right) .
\end{aligned}
$$

which satisfied the last conditions.

It remains to construct the trajectory $\xi(t)$ which verifies:

$$
\begin{aligned}
& \xi\left(t_{i}\right)=\xi_{i}, \xi^{(1)}\left(t_{i}\right)=0, \ldots, \xi^{(5)}\left(t_{i}\right)=0 \\
& \xi\left(t_{f}\right)=\xi_{f}, \xi^{(1)}\left(t_{f}\right)=0, \ldots, \xi^{(5)}\left(t_{f}\right)=0
\end{aligned}
$$
by:

Then, we will get the polynomial of degree 11denoting

$$
\begin{aligned}
& \xi(t)=\xi_{i}+\left(\xi_{f}-\xi_{i}\right) \sigma^{6}(t)(462-1980 \sigma(t)+ \\
& \left.3465 \sigma^{2}(t)-3080 \sigma^{3}(t)+1386 \sigma^{4}(t)-252 \sigma^{5}(t)\right)
\end{aligned}
$$

with, $\sigma(t)=\frac{t_{-} t_{i}}{t_{f}-t_{i}}$

\section{Flatness and Tracking of Trajectory}

By using the expression of the dynamic endogenous feedback calculated in paragraph 4 and the expressions of (32)-(33), we will have the following curly system, with $w_{1} \neq 0$

We have a curly system of order 8 .

Thus, we can choose, if we measure all the state $\left(x_{3}, \dot{x}_{3}, x_{1}, \dot{x}_{1}, \emptyset, \dot{\varnothing}\right)$, the following expressions corresponds of the new controls denoting by:

$$
\begin{aligned}
& \vartheta_{1}=v_{1}^{*}-\sum_{j=0}^{3} k_{1 j}\left(y_{1}^{(j)}-\left(y_{1}^{*}\right)^{(j)}\right) \\
& \vartheta_{2}=v_{2}^{*}-\sum_{j=0}^{3} k_{2 j}\left(y_{2}^{(j)}-\left(y_{2}^{*}\right)^{(j)}\right)
\end{aligned}
$$

$v_{1}^{*}$ and $v_{2}^{*}$ are the inputs of references which corresponds of trajectories of references $y_{1}^{*}$ and $y_{2}^{*}$.

The constant $k_{1 j}$ and $k_{2 j}$ are chosen in order to assure the stability of the systems denoting by:

$$
\begin{aligned}
& e_{1}^{(4)}+k_{13} e_{1}^{(3)}+k_{12} e_{1}^{(2)}+k_{11} e_{1}^{(1)}+k_{10} e_{1}=0 \\
& e_{2}^{(4)}+k_{23} e_{2}^{(3)}+k_{22} e_{2}^{(2)}+k_{21} e_{2}^{(1)}+k_{20} e_{2}=0
\end{aligned}
$$

and,

$$
\begin{aligned}
& \xi_{d}=\xi(t)=\xi_{i}+\left(\xi_{f}-\xi_{i}\right) \cdot \sigma^{6}(t) \cdot(462-1980 \sigma(t)+ \\
& \left.3465 \sigma^{2}(t)-3080 \sigma^{3}(t)+1386 \sigma^{4}(t)-252 \sigma^{5}(t)\right)
\end{aligned}
$$

$\zeta_{d}=\zeta(\xi)=\zeta_{i}+\left(\zeta_{f}-\zeta_{i}\right)\left(\frac{\xi-\xi_{i}}{\xi_{f}-\xi_{i}}\right)\left(9-12\left(\frac{\xi-\xi_{i}}{\xi_{f}-\xi_{i}}\right)+\right.$ $\left.4\left(\frac{\xi-\xi_{i}}{\xi_{f}-\xi_{i}}\right)^{2}\right)$

Finally, we replaced $y_{i}^{(j)}$ and $\left(y_{i}^{*}\right)^{j}$ with their expressions in function of $\left(x_{3}, \dot{x}_{3}, x_{1}, \dot{x}_{1}, \emptyset, \dot{\emptyset}, w_{1}, \dot{w}_{1}\right)$ in order to assure the local exponential convergence of these last to their references.

\section{Simulations}

We present in this part the simulation of the controls and trajectories browsed by the load.

The conditions of simulation can be represented by:

$$
\left\{\begin{array}{l}
\xi=x_{3}+x_{1} \sin \phi \\
\zeta=x_{1} \cos \phi
\end{array}\right.
$$

$x_{3 i}=0$ corresponds to the position of the wagon at $t_{i}$ and $x_{3 f}=10 \mathrm{~m}$ corresponds to the position of the wagon at $t_{f}$. Concerning the length of the cable, we chose at $t_{i}$, $x_{1 i}=-2 m$ and at $t_{f}, x_{1 f}=-1,5 m$, we will have the initial position of the load $\left\{\begin{array}{ll}\xi_{i}= & 0 \\ \zeta_{i} & =-2 m\end{array}\right.$ and the final position is defined by $\left\{\begin{array}{llr}\xi_{f} & =10 \\ \zeta_{f} & =-1,5 m\end{array}\right.$

Figures $2 \mathrm{a}$ and $2 \mathrm{~b}$ show the crane position responses. It is clear that the tracking errors resulting for the two movements (horizontal axis and vertical axis) are acceptable. Figures $3 \mathrm{a}$ and $3 \mathrm{~b}$ represent the corresponding control input based on flatness. Then the law control provides action control values that are suitable for the used actuators. This is yielded from the pole assignment, which imposes convenient dynamics for the closed loop system. Finally, it is obvious that the satisfactory output tracking performance has been achieved through the proposed control scheme. The controls are limited between a minimal intensity and a maximal one. Figure 4 shows the swing of the crane around the vertical and the last figure 
represent the motion of the crane on the phase.

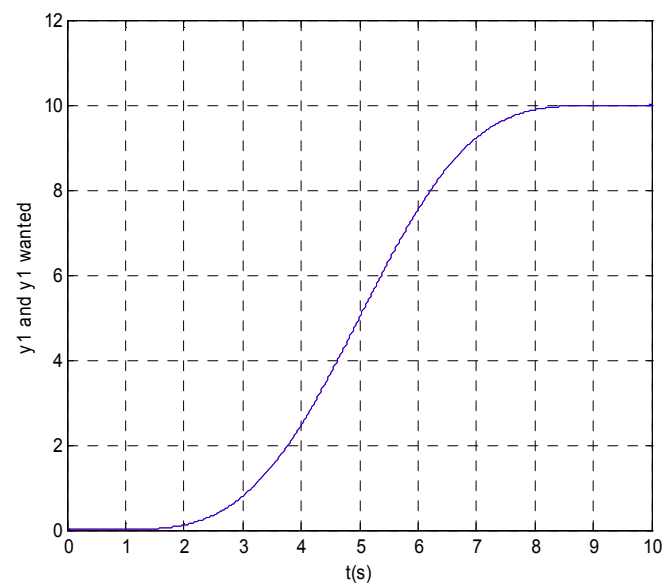

Fig. 2a. The position responses(horizontal axis).

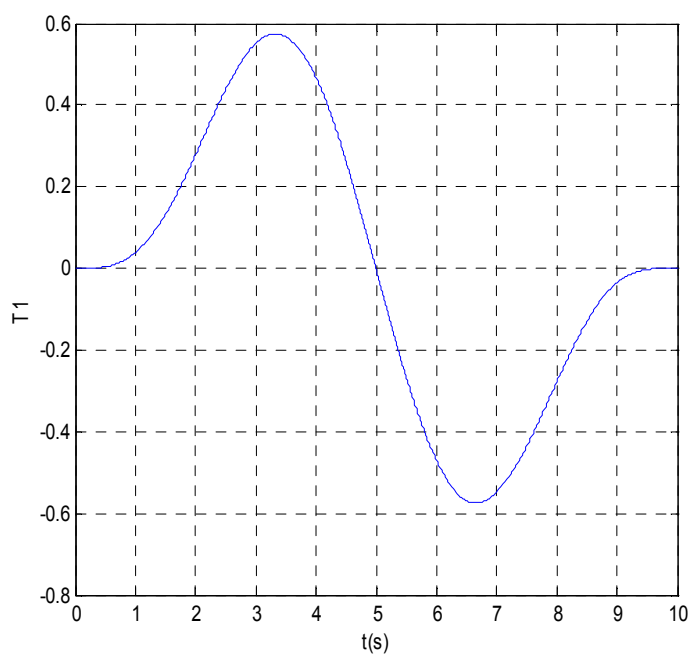

Fig. 3a. The control variable(T1).

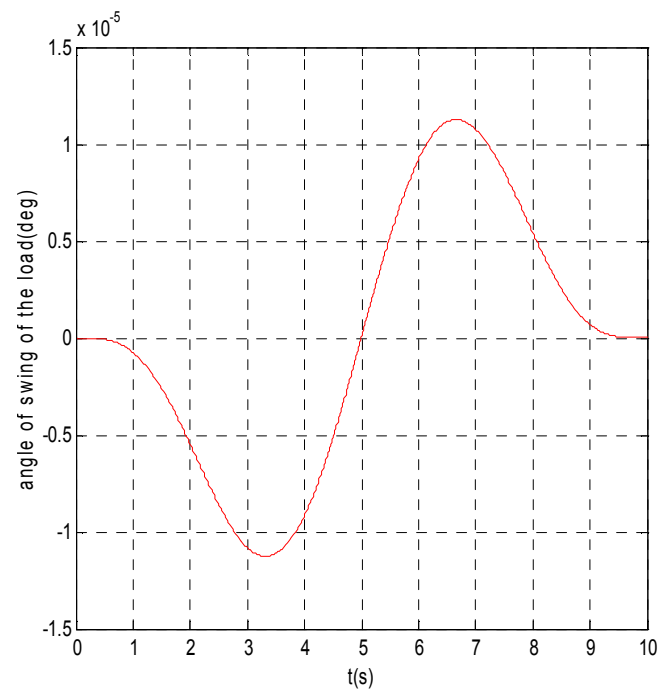

Fig 4. The swing of the crane.

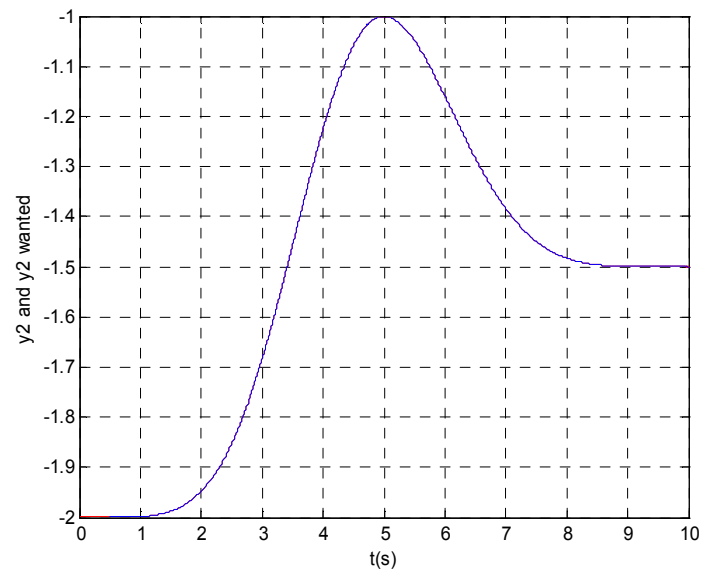

Fig. 2b. The position responses(vertical axis).

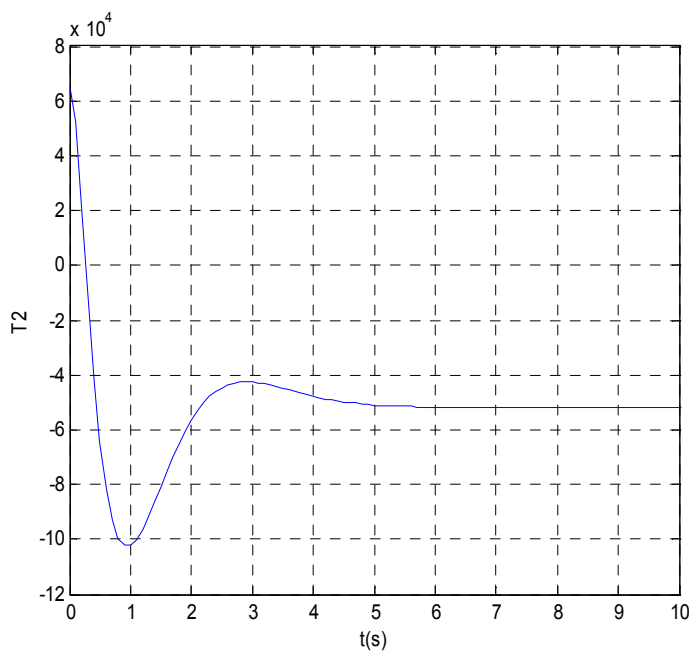

Fig. 3a. The control variable(T2)

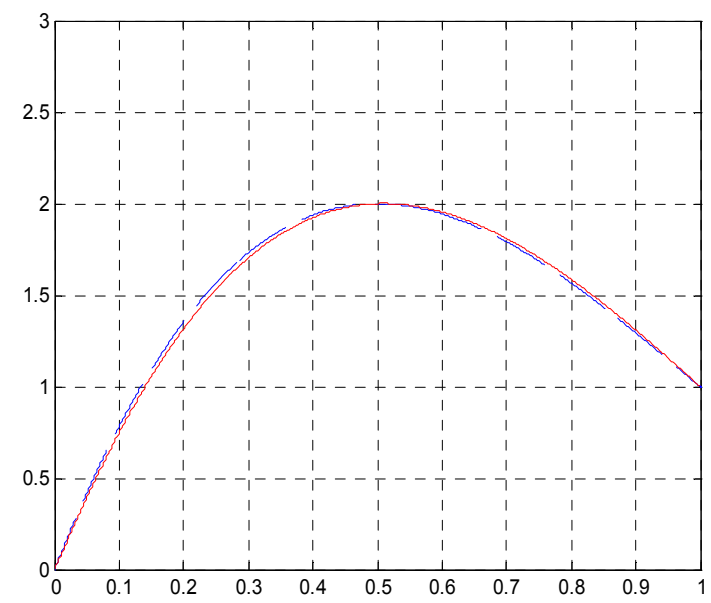

Fig 5. The moving of the crane on the phase. 


\section{Conclusion}

In this paper we are proposed a flatness control of a crane. This control is used to generate the desired trajectory and to force the crane to follow it. Experimental results show the proposed algorithm efficiencies.

The real and the desired moving of the crane indicate that a similar procedure could be applied to controlling the luffing. The problem was also extended to take into account the case when working motion starts with non-zero swing of the payload or there are other disturbances. The swing-free stop control is frequently used in the case of overhead cranes, particularly those intended for transferring large payloads, or in the case of reloading works performed repeatedly. The proposed strategy of controlling the slewing motion used as a whole or only for stopping the swinging payload could increase work safety and improve work quality. Therefore, the objective of the control of the crane by flatness is to increase the productivity and the man's operational security on the one hand and to eliminate the undesirable swings of the load on the other hand.

\section{References}

[1] A. Chelouah, E. Delaleau, P. Martin and P.Rouchon, Differential flatness and control of induction motors, symposium on Control, Optimization and Supervision; Computational engineering in system applications, IMACS Multiconference, pp. 80-85, Lille, 9-12 July 1996.

[2] T. Burg, D. Dawson, C. Rahn, and W. Rhodes."Nonlinear control of an overhead crane via the saturating control approach of Teel". In Proceedings of the Internationl Conference on Robotics and Automation, pages 3155-3160, 1996.

[3] H. Butler, G. Honderd, et J. Van Amerongen." Model reference adaptive of a gantry crane scale model“". IEEE Control system Magazine, pages 57-62, January 1991.

[4] J. Collado, R. Lozano, and I. Fantoni. "Control of a conveycrane based on passivity". In Proceedings of the American Control Conference, pages 1260-1264, 2000.

[5] M. Fliess, J. Lévine, P. Martin, P. Rouchon. "Flatness and defect of non-linear systems": introductory theory and examples, INT. J. Control,1995, Vol. 61, No. 6, 1327-1361.

[6] M. Fliess, J. Lévine, Ph. Martin and P. Rouchon, On differentially flat nonlinear systems, IFAC-Synopsium, NOLCOS'92 pp. 408-412, Borddeaux, 1992.

[7] D. Fragopoulos, M.P. Spathopoulos, and Y. Zheng. "A pendulation control system for offshore lifting operations“". In Proceedings of the 14th IFAC Triennial World Congress, pages 465-470, Beijing, P.R. China, 1999.

[8] T. Gustafsson. "On the design and implementation of a rotary crane controller". European Journal of Control, 2(3):166-175, March 1996.

[9] K. S. Hong, J.H. Kim, et K.I Lee. "Control of a container crane: Fast trversing, and residual sway control from the erspective of controlling an underactuated system". In Proceedings of the American Control Conference, pages 1294-1298, Philadelphia, PA, June 1998. 1-305, 1995.ne: to the crane control system.

[10] J. Lévine, P. Rouchon, G. Yuan, C. Grebogi, B. Hunt, E. Ott, J. Yorke and E. Kostelich, On the control of US navy cranes, European Control Conference, ECC'97, Brussels, July 1997.

[11] Ph. Martin and P. Rouchon, Systèmes plats: planification et suivi de trajectoires, www.math.polytechnique.fr/xups/vo199.

[12] S. C. Martindale, D. M. Dawson, J. Zhu, et C. Rahn. "Approximate nonlinear control for a two degree of freedom overhead crane: Theory and experimentation". In Proceedings of the American Control Conference, pages 301-305, 1995.

[13] K.A.F Moustafa. "Reference trajectory tracking of overhead cranes". Journal of Dynamic Systems, Measurement, and Control, 123:139-141, March 2001.

[14] R. H. Overton. "Anti-sway control system for cantilever cranes". United States Patent, June 1996. Patent No.5, 526,946 .

[15] F. Rotella, F. Carrillo and M. Ayadi, Digital flatness-based robust controller applied to a thermal process, IEEE international Conference on Control application, pp. 936941, Mexico 2001.

[16] R. Rothfuss, J. Rudolph and M. Zeitz, Flatness based control of chemical reactor model, European Control Conference, pp. 637-642, Rome, September 1995.

[17] Y. Sakawa et Y. Shindo. "Optimal control of container cranes“. Automatica, 18(3), 1981, 257-266.

[18] H. Souilem, H. Mekki, N. Derbel, "crane control by flatness", ninth International Multi-Conference on Systems, Signals \& Devices, Chemnitz, Germany, March 2012.

[19] K. Yoshida and H. Kawabe. "A design of saturating control with guaranteed cost and its application to the crane control system". IEEE Transactions on Automatic Control, 37(1):121-127, 1992.

[20] J. Yu, F.L. Lewis, et T. Huang. “ Nonlinear feedback control of a gantry crane“. In Proceeding of the American Control Conference, pages 4310-4315, 1995. 